\title{
A survey of Canadian renal transplant surgeons: Use of ureteric stents and technique of the ureteroneocystotomy
}

Luke F. Reynolds ${ }^{1}$; Tad Kroczak ${ }^{1}$; R. John Honey ${ }^{1}$; Kenneth T. Pace ${ }^{1}$; Jason Y. Lee ${ }^{2}$; Michael Ordon ${ }^{1}$

${ }^{1}$ St Michael's Hospital; ${ }^{2}$ Toronto General Hospital; University of Toronto, Toronto ON, Canada

Cite as: Can Urol Assoc J 2018 June 19; Epub ahead of print. http://dx.doi.org/10.5489/cuaj.5381

Published online June 19, 2018

$* * *$

\section{Abstract}

Introduction: The role of ureteric stenting in renal transplant has been welldemonstrated. The goal of this survey was to determine the utilization of ureteric stents by Canadian transplant surgeons, and how the ureteroneocystotomy and followup is performed.

Methods: An online survey was sent to the 40 surgeon members of the Canadian Society of Transplantation. The primary outcome was the rate of ureteric stent use at the time of renal transplantation. The secondary outcomes were the ureteric stent dwell time, use and type of prophylactic antibiotics, and the use of routine post-transplant ultrasonography. Results: All respondents (25) used ureteric stent routinely and 92\% remove the stent between four and six weeks postoperatively. Prophylactic antibiotics were used $64 \%$ of the time for ureteric stent removal. The majority of surgeons do not routinely perform a post-stent removal ultrasound. Fifty-six percent of respondents perform a refluxing anastomosis.

Conclusions: Ureteric stents are routinely used in renal transplant in Canada. Areas for improvement and topics of debate identified from this survey are the need for peri-stent removal antibiotics, the role of post-stent removal ultrasound, the duration of stent dwell time, and the need for a non-refluxing ureteroneocystotomy. 


\section{Introduction}

In 2016 there were a reported 1731 renal transplants performed in Canada. ${ }^{1}$ Although transplantation is an effective alternative to renal replacement therapy, it is associated with a number of significant surgical complications that are often associated with the ureteroneocystostomy (UNC). ${ }^{2}$ In order to mitigate some of the early complications such as urine leak, urinary obstruction, and ureterovesical stricture, ureteric stents can be used. Meta-analyses have demonstrated that ureteric stenting vs. no stent results in a decrease of major urologic complications following transplantation. ${ }^{3,4}$ Although routine stenting appears to be of benefit, no Canadian guidelines exist for the use, type, or duration of ureteral stents and the role for peri-stent removal antibiotics is not clear. Although ureteral stents are used commonly in reconstructive urology, many transplant surgeons in Canada are not urologists.

Another contentious issue is the need for a non-refluxing ureteroneocystostomy (UNC). Various techniques exist for performing the UNC which can be divided into intravesical vs. extravesical and refluxing vs. non-refluxing anastomoses. Compared to the traditional non-refluxing Politano-Leadbetter technique, the Lich-Gregoir technique results in decreased complications. ${ }^{5}$ A more recently described technique that uses a refluxing anastomosis appears to have comparable surgical outcomes compared to nonrefluxing anastomoses. ${ }^{6}$

Urologists, general surgeons and vascular surgeons are all specialists that are involved in renal transplantation in Canada. The goal of this study was to describe practice patterns among the various trained specialists performing renal transplantation. Specifically, we wanted to assess (1) the demographics of Canadian renal transplant surgeons, (2) how often ureteric stents are utilized, (3) how the UNC is performed, and (4) post-operative antibiotic use and surveillance ultrasound.

\section{Methods}

This study used an online survey sent to surgeon members of the Canadian Society of Transplantation involved in kidney transplantation. Research Ethics Board approval was received from St. Michael’s Hospital, Toronto, Ontario, Canada.

The inclusion for this study was any practitioner from any specialty provided they are actively participating in renal transplantation as the primary surgeon. The survey was sent to participants by email in the summer of 2016 and the survey was closed in November of 2016. Participation was voluntary. A total of 40 surgeons were contacted to participate in the survey.

Data integrity was maintained throughout the study. Logic rules within the database were used to maximize integrity and random data validation checks were performed by a research coordinator. 
The primary outcome was the rate of ureteric stent use at the time of renal transplantation. The secondary outcomes were the ureteric stent dwell time, use and type of prophylactic antibiotics and the use of routine post-transplant ultrasonography (US).

\section{Results}

Of the 40 surgeons that were contacted, a total of 25 responded to the online survey (62.5\%) with $64 \%$ of them practicing in Ontario. The majority of surgeons (88\%) had $>5 \mathrm{yrs}$ of renal transplantation experience with $24 \%$ of respondents reporting an average annual volume of $>50$ renal transplants per year; only $8 \%$ reporting $<20$ cases per year. The majority of transplant surgeons that responded were urologists (64\%), while the rest were made up of general surgeons and vascular surgeons (Figure 1). Fellowship training in renal transplantation was reported by $62.5 \%$ of respondents.

Ureteric stents were used universally with $100 \%$ of surgeons reporting routine stent use. Most respondents (92\%) reported removing the stent 4-6 weeks post-transplant. Oral antibiotics were administered for stent removal $64 \%$ of the time: $38 \%$ gave a single dose around the time of stent removal and 16\% prescribed antibiotics for greater than 2 days. Ciprofloxacin was the most common drug administered (10/16) followed by trimethoprim-sulfamethoxazole (5/16), with one respondent reporting the use of cefixime at the time of stent removal.

Only 28\% reported the use of routine post-stent removal allograft US and the majority of those surgeons performed an US at 4-6 weeks post-stent removal. Respondents were almost equally divided on the use of refluxing (56\%) and nonrefluxing (44\%) ureteric anastomotic techniques. The proportion of Urologists (7/16) and General Surgeons (3/7) who performed non-refluxing anastomoses were similar. The catheter was removed $20 \%$ of the time on both post-operative day 3 and 4 and $56 \%$ of the time on post-operative day 5 (figure 2).

\section{Discussion}

Renal transplantation liberates patients from the burden of dialysis and is the most cost effective and definitive treatment for end stage renal failure. ${ }^{7,8}$ It is without question the preferred treatment for patients with ESRD in Canada. The role of the urologist in Canada in renal transplantation continues to be an important one. In this survey the majority of surgeons performing renal transplants come from a urologic surgery background, with urologists and general surgeons performing the bulk of renal transplants in Canada.

Based on the results of this survey, ureteral stents are placed routinely by all surgeons in Canada performing renal transplantation. While almost all respondents reported removing ureteral stents between 4-6 weeks post-operatively, emerging evidence is suggestive that early stent removal is safe and may be beneficial. One area of improvement identified in this survey may be the utilization of antibiotic prophylaxis 
around the time of stent removal. The AUA guidelines on antibiotic prophylaxis do recommend the use of prophylactic antibiotics in the setting of a cystoscopic stent removal. ${ }^{9}$ Peri-stent removal antibiotic coverage in accordance with local patterns of ureteral stent colonization seems prudent. Among respondents, no obvious preference exists for refluxing versus non-refluxing anastomoses, which likely reflects the lack of clear evidence of superiority of technique. The utility of routine post-stent removal US is also a topic for further investigation, as less than a $3^{\text {rd }}$ of respondents reported the use of routine US.

As illustrated by the results of this survey, ureteric stenting has become routine during renal transplantation. The evidence for routine prophylactic stenting over no stents is convincing. In one meta-analysis that included 49 studies (5 randomized and 44 case series), a significant decrease in complications was observed with prophylactic stenting. Rates of obstruction/stricture, leak/necrosis and significant hematuria were decreased from $9.0 \%$ to $1.5 \%$ with prophylactic stents using data from the randomized trials. ${ }^{3} \mathrm{~A}$ second meta-analysis that included 2 additional randomized studies $(7$ total) produced results similar to the meta-analysis by Mangus et al. In this study, the authors demonstrated that major urologic complications defined as urine leak or obstruction was significantly reduced (RR 0.24) with stent use. Another significant finding in this review is that increased surgeon volume was associated with less complications. ${ }^{4}$

There appears to be a clear advantage to prophylactic stenting versus no stent for both live and deceased donors. What is less clear is if prophylactic stenting is superior to stenting in an on-demand fashion for extravesical anastamoses. No prospective randomized trials have compared prophylactic versus selective stenting. In a retrospective study that had overall low rates of complications in both groups, and controlled for sex and allograft type, prophylactic stenting resulted in less ureterovesical complications compared to using stents at the discretion of the surgeon (OR 0.30; $\mathrm{p}=0.009$ ) ${ }^{10}$

The drawback of routine utilization of ureteric stents in renal transplantation is the need for an ancillary procedure (cystoscopic removal), pain, bacterial colonization and urinary tract infection (UTI), hematuria, and the possibility of stent encrustation and migration. Colonization of the stent in the immunosuppressed urinary system is an obvious concern in the post-operative period as this can lead to serious infection and potential graft loss. Rates of culture proven infection in the post-transplant period can be as high as $73 \%{ }^{11,12}$ Wilson et al, demonstrated an increased risk of UTI with stent use (RR 1.49); the studies included in this review include patients who were on antibiotic prophylaxis for pneumocystis jiroveci pneumonia. ${ }^{4}$ In a prospective series that assessed stent colonization and UTIs with stents of varying time periods, a total of $22.4 \%$ of ureteral stents were colonized and $7.4 \%$ of patients developed a UTI while on cotrimoxazole. Stents that were left in for 5 weeks had the highest rate of colonization. ${ }^{13}$ The high rates of colonization and infection while on antibiotic prophylaxis raises the 
concern for co-trimoxazole resistant bacterial colonization. In our survey the vast majority of stents were left in for 4-6 weeks prior to being removed.

A Cochrane review published in 2018 that included 5 randomized controlled trials or quasi-randomized controlled trials assessed early versus late ureteric stent removal in renal transplant. The results of this study did not demonstrate a clear risk of stent removal before the third week post-operatively. There were no significant differences in the incidence of major urologic complications such as urinary obstruction, leak, fistula or stenosis (RR 1.87 CI 0.61-5.71). ${ }^{12}$ Other important benefits of early stent removal include improved quality of life after 6 weeks, ${ }^{14}$ less lower urinary tract symptoms, ${ }^{15}$ improved cost-effectiveness. ${ }^{16}$ Accordingly, based this data and the results of our survey efforts need to be made to consider removing stents earlier to alleviate the potential negative effects of stents.

A more debated topic is the optimal method of performing the UNC and the need for the anti-reflux mechanism. Various techniques have been described to acquire continuity of the transplanted urinary system. The most studied and commonly used techniques are the intravesical non-refluxing Politano-Leadbetter, ${ }^{17}$ extravesical nonrefluxing Lich-Gregoir, ${ }^{18,19}$ the U-stitch, ${ }^{20}$ and a refluxing extravesical anastomosis described by Starzl. ${ }^{21}$ A recent review has demonstrated that the Lich-Gregoir has less urine leak events (RR 0.47 CI 0.30-0.75), a decreased stricture rate (RR 0.55, CI 0.390.76 ) and decreased rates of hematuria (RR $0.28 \mathrm{CI} 0.16-0.49$ ) compared to the Politano-Leadbetter technique. ${ }^{5}$ A meta-analysis of 6 articles comparing ureteroureterostomy to UNC (Lich-Gregoir) showed no difference in overall complication rates. ${ }^{22}$ The ureteroureterostomy provides a nice option for complex reoperations, management of a short donor ureter, avoids a cystotomy, allows for earlier catheter removal, preserves the anti-reflux mechanism, and allows access to the ureter in a retrograde fashion that is often not possible with a UNC.

A modification of the Lich-Gregoir technique coined the "full-thickness" anastomosis is performed by spatulating the ureter, creating a cystotomy followed by a full thickness anastomosis of the bladder to the ureter. ${ }^{23}$ This anastomosis differs from the Lich-Gregoir in that the detrusor is not closed over the ureter, resulting in a less technically challenging and refluxing anastomosis. A recent study by Kayler et al. compared full-thickness versus the Lich-Gregoir techniques and found no significant differences in overall complications (strictures, obstruction, urine leak, urinary tract infections, graft function) at one year. ${ }^{6}$ In this study, ureteral stents were used much more commonly in the Lich-Gregoir cohort $(\mathrm{P}<0.01)$. The use of this full thickness technique raises the question, are non-refluxing anastomoses necessary? While most patients are asymptomatic from reflux, there are concerns of the effect of reflux on renal function and increased risk of urinary tract infections in an immunocompromised urinary system. Based on retrospective data from numerous studies, vesicoureteral reflux does appear to 
be safe in renal transplant. ${ }^{24-27}$ Interestingly a very large proportion (40-61\%)of patients who have a non-refluxing anastomosis actually do have reflux. ${ }^{24-26}$

The results of our study must be interpreted within the context of our study limitations. This was a survey study and as such is at risk for sampling and recall bias. Also, in assessing the use of post-operative surveillance US although the surgeon may not routinely use post-stent removal US, the transplant nephrologists that follow these patients longitudinally may be performing this routinely without the surgeon's knowledge. In addition, since some respondents may not be the surgeon who removes the ureteric stent, their knowledge of practices around and after stent removal may be inaccurate and as such impact the data on this topic.

\section{Conclusion}

In conclusion the results from this survey demonstrate that stent utilization for Canadian kidney transplant surgeons is standard. Variation in practice is seen with peri-stent removal antibiotics, post-stent removal US, and the use of a non-refluxing UNC. Based on emerging evidence, Canadian transplant patients may benefit from earlier stent removal and more rigorous prophylaxis at the time of stent removal. 


\section{Reference}

1. CORR Annual Statistics, 2007 to 2016. Canadian Institute for Health Information; https://www.cihi.ca/en/corr-annual-statistics-2007-to-2016. Accessed March 28, 2018.

2. Kumar A, Verma BS, Srivastava A, Bhandari M, Gupta A, Sharma R. Evaluation of the urological complications of living related renal transplantation at a single center during the last 10 years: impact of the Double-J* stent. J Urol. 2000;164(3 Pt 1):657-660.

3. Mangus RS, Haag BW. Stented versus nonstented extravesical ureteroneocystostomy in renal transplantation: a metaanalysis. Am J Transplant. 2004;4(11):1889-1896.

4. Wilson CH, Bhatti AA, Rix DA, Manas DM. Routine intraoperative stenting for renal transplant recipients. Transplantation. 2005;80(7):877-882.

5. Alberts VP, Idu MM, Legemate DA, Laguna Pes MP, Minnee RC. Ureterovesical anastomotic techniques for kidney transplantation: a systematic review and metaanalysis. Transpl Int. 2014;27(6):593-605.

6. Kayler L, Zendejas I, Molmenti E, Chordia P, Schain D, Magliocca J. Kidney transplant ureteroneocystostomy: comparison of full-thickness vs. Lich-Gregoir techniques. Clin Transplant. 2012;26(4):E372-380.

7. Laupacis A, Keown P, Pus N, et al. A study of the quality of life and cost-utility of renal transplantation. Kidney Int. 1996;50(1):235-242.

8. Meier-Kriesche HU, Ojo AO, Port FK, Arndorfer JA, Cibrik DM, Kaplan B. Survival improvement among patients with end-stage renal disease: trends over time for transplant recipients and wait-listed patients. J Am Soc Nephrol. 2001;12(6):1293-1296.

9. Wolf JS, Jr., Bennett CJ, Dmochowski RR, et al. Best practice policy statement on urologic surgery antimicrobial prophylaxis. J Urol. 2008;179(4):1379-1390.

10. Ordon M, Ghiculete D, Stewart R, Pace KT, Honey RJ. The role of prophylactic versus selective ureteric stenting in kidney transplant patients: a retrospective review. Prog Transplant. 2014;24(4):322-327.

11. Ranganathan M, Akbar M, Ilham MA, Chavez R, Kumar N, Asderakis A. Infective complications associated with ureteral stents in renal transplant recipients. Transplant Proc. 2009;41(1):162-164.

12. Thompson ER, Hosgood SA, Nicholson ML, Wilson CH. Early versus late ureteric stent removal after kidney transplantation. Cochrane Database Syst Rev. 2018;1:CD011455. 
13. Sarier M, Demir M, Duman I, Yuksel Y, Demirbas A. Evaluation of Ureteral Stent Colonization in Live-Donor Renal Transplant Recipients. Transplant Proc. 2017;49(3):415-419.

14. Patel P, Rebollo-Mesa I, Ryan E, et al. Prophylactic Ureteric Stents in Renal Transplant Recipients: A Multicenter Randomized Controlled Trial of Early Versus Late Removal. Am J Transplant. 2017;17(8):2129-2138.

15. Huang L, Wang X, Ma Y, et al. A comparative study of 3-week and 6-week duration of double-J stent placement in renal transplant recipients. Urol Int. 2012;89(1):89-92.

16. Parapiboon W, Ingsathit A, Disthabanchong S, et al. Impact of early ureteric stent removal and cost-benefit analysis in kidney transplant recipients: results of a randomized controlled study. Transplant Proc. 2012;44(3):737-739.

17. Politano VA, Leadbetter WF. An operative technique for the correction of vesicoureteral reflux. J Urol. 1958;79(6):932-941.

18. Gregoir W. [Congenital vesico-ureteral reflux]. Acta Urol Belg. 1962;30:286-300.

19. Lich R, Jr., Howerton LW, Davis LA. Childhood urosepsis. J Ky Med Assoc. 1961;59:1177-1179.

20. Taguchi Y, Klauber GT, MacKinnon KJ. Implantation of transplant ureters: a technique. J Urol. 1971;105(2):194-195.

21. Starzl TE, Shapiro R, Tzakis A, Hakala TR. A new technique of extravesical ureteroneocystostomy for renal transplantation. Transplant Proc. 1989;21(5):3856-3858.

22. Suttle T, Fumo D, Baghmanli Z, Saltzman B, Ortiz J. Comparison of Urologic Complications Between Ureteroneocystostomy and Ureteroureterostomy in Renal Transplant: A Meta-Analysis. Exp Clin Transplant. 2016;14(3):276-281.

23. Kayler L, Kang D, Molmenti E, Howard R. Kidney transplant ureteroneocystostomy techniques and complications: review of the literature. Transplant Proc. 2010;42(5):1413-1420.

24. Margreiter M, Gyori GP, Bohmig GA, Trubel S, Muhlbacher F, Steininger R. Value of routine voiding cystourethrography after renal transplantation. Am J Transplant. 2013;13(1):130-135.

25. Jung GO, Chun JM, Park JB, et al. Clinical significance of posttransplantation vesicoureteral reflux during short-term period after kidney transplantation. Transplant Proc. 2008;40(7):2339-2341.

26. Favi E, Spagnoletti G, Valentini AL, et al. Long-term clinical impact of vesicoureteral reflux in kidney transplantation. Transplant Proc. 2009;41(4):1218-1220. 
27. Vianello A, Pignata G, Caldato C, et al. Vesicoureteral reflux after kidney transplantation: clinical significance in the medium to long-term. Clin Nephrol. 1997;47(6):356-361. 
Figures and Tables

Fig. 1. Primary discipline of training.

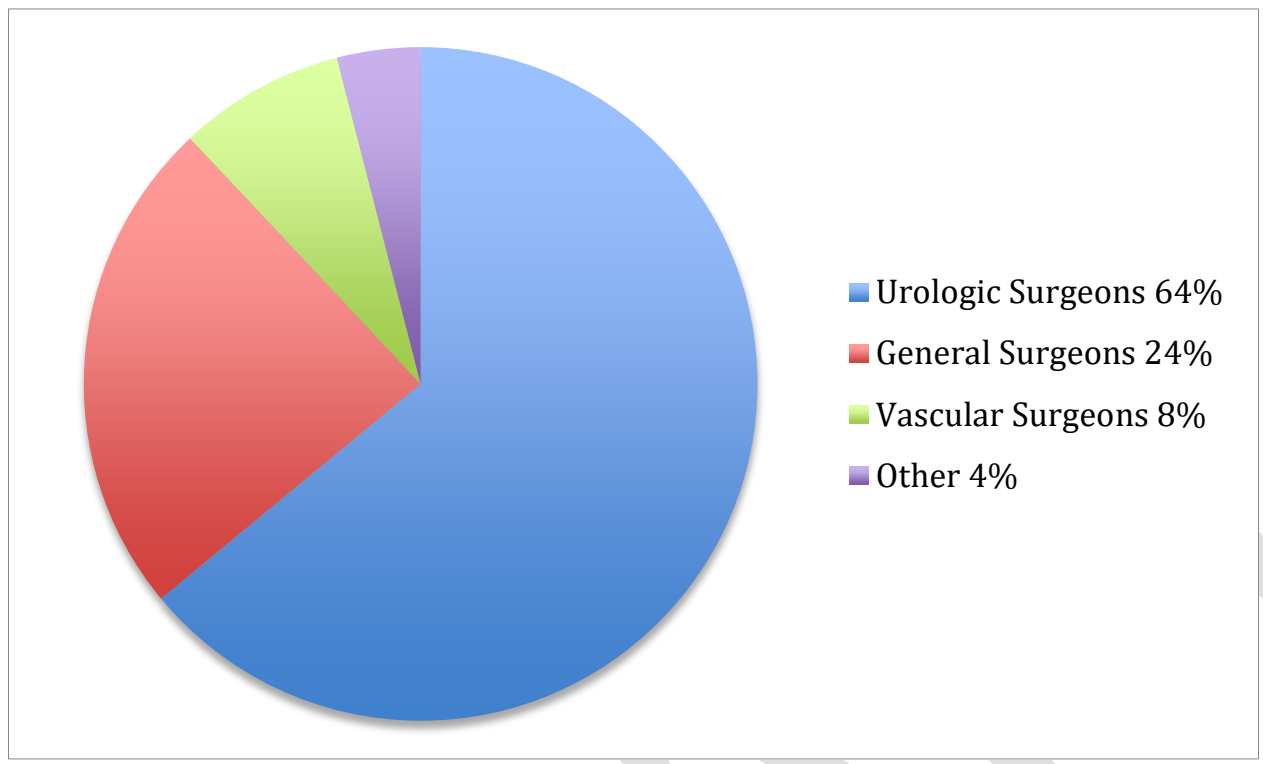

Fig. 2. Postoperative day of Foley catheter removal.

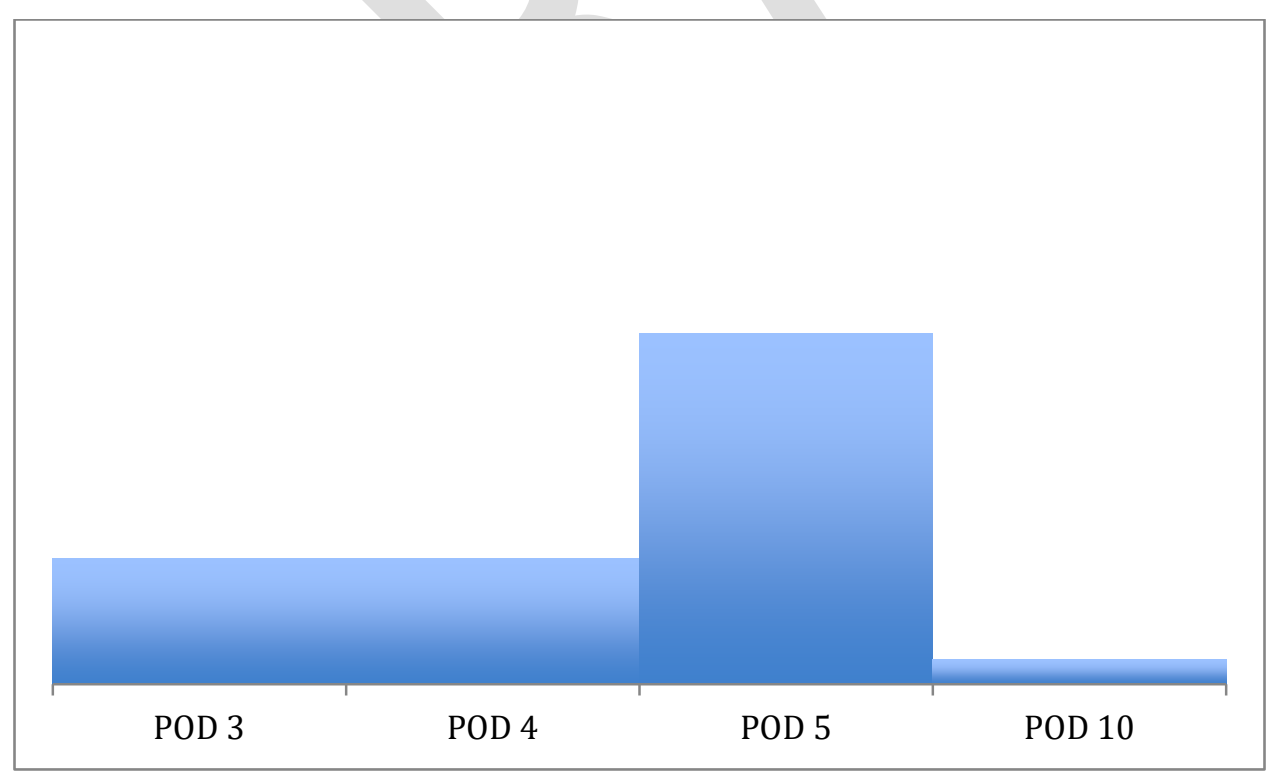

